\title{
A comparison of conventional methods for the quantification of bacterial cells after exposure to metal oxide nanoparticles
}

\author{
Hongmiao Pan ${ }^{1}$, Yongbin Zhang ${ }^{2}$, Gui-Xin He ${ }^{3}$, Namrata Katagori ${ }^{3}$ and Huizhong Chen ${ }^{1 *}$
}

\begin{abstract}
Background: Due to potential interference of nanoparticles on bacterial quantification, there is a challenge to develop a fast, accurate and reproducible method for bacterial quantification. Currently various bacterial quantification methods are used by researchers performing nanoparticles study, but there has been no efficacy evaluation of these methods. Here we study interference of nanoparticles on three most commonly used conventional bacterial quantification methods, including colony counting to determine the colony-forming units (CFU), spectrophotometer method of optical density (OD) measurement, and flow cytometry (FCM).

Results: Three oxide nanoparticles including $\mathrm{ZnO}, \mathrm{TiO}_{2}$, and $\mathrm{SiO}_{2}$ and four bacterial species including Salmonella enterica serovar Newport, Staphylococcus epidermidis, Enterococcus faecalis, and Escherichia coli were included in the test. Results showed that there is no apparent interference of the oxide nanoparticles on quantifications of all four bacterial species by FCM measurement; CFU counting is time consuming, less accurate and not suitable for automation; and the spectrophotometer method using OD measurement was the most unreliable method to quantify and detect the bacteria in the presence of the nanoparticles.
\end{abstract}

Conclusion: In summary, FCM measurement proved to be the best method, which is suitable for rapid, accurate and automatic detection of bacteria in the presence of the nanoparticles.

Keywords: Nanoparticles, Bacterial quantification, Flow cytometry (FCM), Colony-forming units (CFU), Optical density (OD)

\section{Background}

Nanoparticles (NPs) offer spectacular properties to their bulk materials, such as a high surface area to volume ratio, new mechanical, chemical, electrical, optical, magnetic, electro-optical, and magneto-optical properties [1]. Nanotechnology is one of the fastest growing areas of the high tech economy [2,3]. Products using nanoparticles - also known as nanomaterials (particle sizes less than $100 \mathrm{~nm}$ )-can be found in almost every area of our daily lives, from cosmetics to clothing to foods to drug products [4-7]. There are hundreds of cosmetics that contain nanomaterials, such as $\mathrm{ZnO}, \mathrm{TiO}_{2}$, and $\mathrm{SiO}_{2}$, in

\footnotetext{
* Correspondence: huizhong.chen@fda.hhs.gov

'Division of Microbiology, National Center for Toxicological Research, U.S. Food and Drug Administration, Jefferson, AR 72079, USA

Full list of author information is available at the end of the article
}

the market now and the number of these products are increasing rapidly [8]. Nanoscale materials can find use in many areas related to the food industry including agriculture, food processing, food security, packaging, nutrition and neutraceuticals [9-11]. Nanoscale materials have been used as novel antimicrobial agents [12]. Due to their powerful antimicrobial activity and particular modes of action, nanoparticles provide an attractive alternative to classic antibiotics in the development of next-generation antibiotic agents [13-15]. Various antimicrobial nanoparticles and nanosized carriers for antibiotics delivery have been developed to effectively treat infectious diseases, especially those caused by antibioticresistant microorganisms [16,17]. Nanoparticles behave differently from their respective bulk materials and thus 
the unique properties of the nanoparticles might also cause adverse health effects on human, animal and environment. The speedy commercialization of nanotechnology requires thoughtful and careful environmental, animal and human health safety assessment $[18,19]$.

The detection and quantification of viable bacteria plays a critical role in quality control programs of the food, cosmetics and drug industry to prevent illness and in clinical diagnosis and therapeutics. Currently there are many methods used for the detection and quantification of bacteria, ncluding conventional and molecular approaches [20-24]. Conventionally identification of bacteria is usually performed by three methods including culture-based counting for colony-forming units (CFU) [22,25], spectrophotometer method of optical density (OD) measurement [23,24], and flow cytometry (FCM) [26,27]. In spite of the sensitivity and reliability, counting CFU is time-consuming and labor-intensive $[28,29]$. CFU determination is the conventional method to quantify bacteria, but only detects those that are able to grow on specific solid media, which excludes the detection of unculturable live, inactive or damaged bacterial cells $[30,31]$. Therefore, CFU counting tends to undercount the actual number of the bacteria. For example, anaerobic bacteria are not able to grow on the media and cultural conditions suitable for growth of aerobic bacteria. Optical density method measures turbidity associated directly with bacterial growth which is rapid, low cost and nondestructive, however, it measures live as well as dead bacterial cell debris. Flow cytometry is a relatively newly developed technique and enables a fast and reliable detection of all bacteria including the non-cultivable microorganisms. It enables researchers to reliably distinguish and quantitate live and dead bacteria with the aid of a flow cytometer in a mixed population containing various bacterial types [32]. Besides, Flow cytometry method is able to provide morphometric and functional properties of the detected bacteria [33,34]. Currently all these three methods are employed to quantify bacterial contents in the presence of nanoparticles [35-39]. So far there has not been any research performed concerning potential interference by nanoparticles on the bacterial counting methods

The aim of this study was to compare three commonly used conventional methods for bacterial detection and quantification in the presence of nanoparticles. In this study, three nanoparticles, $\mathrm{ZnO}, \mathrm{TiO}_{2}$, and $\mathrm{SiO}_{2}$, which are commonly used in commercial products and four important human pathogens (Salmonella enterica serovar Newport, Staphylococcus epidermidis, Enterococcus faecalis, and Escherichia coli) frequently found in various products and environment representing Gram-positive and Gram-negative bacteria were employed for this study.

\section{Results and discussion}

\section{Physical and chemical characterizations of nanomaterials}

It is critical to conduct physical and chemical characterization of testing nanomaterials in nanotechnology research. Size, size distribution, surface charge, aggregation or agglomeration status, and shape have been considered as the most important parameters for nanomaterials. We evaluated these parameters using TEM and Zetasizer as described in the material and methods section. TEM analysis indicated that the $\mathrm{ZnO}, \mathrm{TiO}_{2}$ and $\mathrm{SiO}_{2}$ nanoparticles have spherical shape with slightly agglomeration (Figure 1 ). The primary size of $\mathrm{ZnO}, \mathrm{TiO}_{2}$ and $\mathrm{SiO}_{2}$ nanoparticles were measured as $14.0 \pm 4.9 \mathrm{~nm}, 19.7 \pm 5.7 \mathrm{~nm}$ and $17.4 \pm 5.1 \mathrm{~nm}$, respectively (Table 1 ). The range of the diameter of the $\mathrm{ZnO}$, $\mathrm{TiO}_{2}$ and $\mathrm{SiO}_{2}$ nanoparticle was 6.3-30.5 nm, 10.2$31.2 \mathrm{~nm}$ and 8.0-27.9 $\mathrm{nm}$. Zetasizer analysis indicated that the average size of $\mathrm{ZnO}, \mathrm{TiO}_{2}$ and $\mathrm{SiO}_{2}$ nanoparticles in buffer solution was $2308.3 \pm 159.1 \mathrm{~nm}, 2738.3 \pm$ $303.3 \mathrm{~nm}$ and $915.0 \pm 35.8 \mathrm{~nm}$ (mean $\pm \mathrm{SD}$ ). The average surface charge of the $\mathrm{ZnO}, \mathrm{TiO}_{2}, \mathrm{SiO}_{2}$ nanoparticles in buffer solution was $17.6 \pm 0.7 \mathrm{mV}, 27.2 \pm 3.1 \mathrm{mV},-5.7 \pm$ $0.4 \mathrm{mV}$, respectively (Table 1 ). TEM directly measured the primary size of the nanoparticles based on the projected area; while Dynamic Light Scattering (DLS) measured the hydrodynamic diameter of the nanoparticles based on the translational diffusion area of the particle being measured. The same samples of these nanoparticles in buffer were measured with a bigger size by zetasizer analysis than the measurement using TEM. This is due to the differences in the weighted averages determined by these two techniques, and also the differences in the physical properties measured. TEM is sensitive to the size of primary particles, whereas DLS is sensitive to the presence of small quantities of large particles or aggregates.

\section{Effect of concentrations of nanoparticle on quantification of bacteria}

Potential interference of nanoparticles on the quantification of bacteria poses a challenge for the detection of bacterial contaminations in various consumer products. Therefore, development of a rapid, sensitive and accurate method for detection of bacteria in the presence of nanoparticles is crucial for food, drug, cosmetic and other consumable products. Among many bacterial identification and quantification methods, three of them including culture-based counting for CFU, spectrophotometer method of optical density measurement, and more recently flow cytometry are commonly used. $\mathrm{ZnO}, \mathrm{TiO}_{2}$, and $\mathrm{SiO}_{2}$ have been found in many commercial products including food, food supplements, cosmetics and drugs. S. enterica Newport, S. epidermidis, E. faecalis, and E. coli, which are important human pathogens, are good representatives for Gram-positive and Gram-negative bacteria (Table 2). In this experiment the effect of 
Figure 1 Characterization of $\mathrm{ZnO}, \mathrm{TiO}_{2}$, or $\mathrm{SiO}_{2}$ nanoparticles by transmission electron microscopy (TEM). Nanoparticles were deposited on formvar carbon coated grids and dried for TEM imaging. Images were analyzed in high resolution mode with an acceleration voltage of $100 \mathrm{kV}$. Morphology of $\mathrm{ZnO}, \mathrm{TiO}_{2}$ or $\mathrm{SiO}_{2}$ is shown in left, middle and right of the above images. Scale Bar $=20 \mathrm{~nm}$.

various concentrations of nanoparticles on quantification of S. enterica Newport, S. epidermidis, E. faecalis, and $E$. coli was investigated by exposing $5 \mathrm{ml}$ of samples containing approximately $10^{9}$ cells $/ \mathrm{ml}$ to various concentrations of $\mathrm{ZnO}, \mathrm{TiO}_{2}$, and $\mathrm{SiO}_{2}(0,0.1,0.2,0.3,0.5$, and $1 \mathrm{mg} /$ $\mathrm{ml}$ final concentration) for $1 \mathrm{hr}$, respectively (Table 3). As shown in Table 3, with increasing concentrations of $\mathrm{ZnO}$, $\mathrm{TiO}_{2}$, and $\mathrm{SiO}_{2}$, there was no apparent interference of the nanoparticles on quantifications of all four bacterial species by flow cytometry measurement using the BacLight LIVE/ DEAD bacterial viability and counting kit. As shown in Figure 2 as example, two distinctive groups were formed. Group P2 was the population of living bacterial cells, while group P3 was the population of dead bacterial cells at the presence of $0.2 \mathrm{mg} / \mathrm{ml}$ nanoparticles. Compared to a control, which did not contain nanoparticles, no shifts of the bacterial population or background increase were observed (Figure 2). Since more than 20,000 bacterial cells per sample were counted by flow cytometry measurement, high accuracy and excellent reproducibility of the quantification was achieved for both live and dead bacterial cells (Table 3). Although no apparent interference of the nanoparticles on quantifications of all four bacterial species was observed by using CFU counting, it was a time consuming and labor intensive procedure. Besides, it took long time training and practice for mastering the technique of dilution in order to get reliable counts from one batch to another and from one plate to another in CFU counting. Furthermore, the data obtained by CFU measurement is less accurate and reproducible due to a limited number of bacterial cells counted (several hundred bacterial colonies counted (Table 3). The decreasing numbers of

Table 1 Characterization of $\mathrm{TiO}_{2}, \mathrm{ZnO}$, and $\mathrm{SiO}_{2}$ nanoparticles in Milli- $Q$ water solutions

\begin{tabular}{llll}
\hline Physical Parameters & $\mathrm{ZnO}$ & $\mathrm{TiO}_{\mathbf{2}}$ & $\mathrm{SiO}_{\mathbf{2}}$ \\
\hline Primary size $(\mathrm{nm})$ & $14.0 \pm 4.9$ & $19.7 \pm 5.7$ & $17.4 \pm 5.1$ \\
Primary size range $(\mathrm{nm})$ & $6.3-30.5$ & $10.2-31.2$ & $8.0-27.9$ \\
Hydrodynamic size $(\mathrm{nm})$ & $2738.3 \pm 303.3$ & $2308.3 \pm 159.1$ & $915.0 \pm 35.8$ \\
Shape & spherical & spherical & spherical \\
Agglomerate in solution & Yes & Yes & Yes \\
Zeta potential $\zeta(\mathrm{mV})$ & $17.6 \pm 0.7$ & $27.2 \pm 3.1$ & $-5.7 \pm 0.4$ \\
\hline
\end{tabular}


Table 2 Bacterial species used in the study

\begin{tabular}{|c|c|c|c|}
\hline Species name & Gram $^{1}$ & $\begin{array}{l}\text { Culture } \\
\text { condition }\end{array}$ & Isolation \\
\hline $\begin{array}{l}\text { Salmonella enterica serovar } \\
\text { Newport }\end{array}$ & - & aerobic & human intestine \\
\hline $\begin{array}{l}\text { Staphylococcus epidermidis } \\
\text { ATCC } 12228\end{array}$ & + & aerobic & human skin \\
\hline Enterococcus faecalis ATCC 27274 & + & anaerobic & human intestine \\
\hline Escherichia coli ATCC 25922 & - & anaerobic & human intestine \\
\hline
\end{tabular}

${ }^{1}+$, Gram-positive; -, Gram-negative.

the bacteria by using CFU and flow cytometry were resulted from antibacterial effects caused by both nanoparticles $\mathrm{TiO}_{2}$ and $\mathrm{ZnO}$. As shown in Table 3, nanoparticles had adverse effect on quantification of bacteria using the spectrophotometer method of optical density measurement with severity of $\mathrm{TiO}_{2}>\mathrm{ZnO}>\mathrm{SiO}_{2}$. For example, in the presence of $0.1 \mathrm{mg} \mathrm{TiO}_{2}$, the number of $S$. epidermidis cells was not detectable due to high background interference from the nanoparticles in the samples. With $0.3 \mathrm{mg} / \mathrm{ml}$ $\mathrm{TiO}_{2}$, S. enterica Newport and E. faecalis cells were 45.2 and $42.8 \%$ of those measured by FCA, respectively. On the other hand, E. coli cells were more than 8 -folds than that by FCA in the presence of $0.3 \mathrm{mg} / \mathrm{ml} \mathrm{TiO}_{2}$.

\section{Effect of bacterial concentrations on quantification of bacteria after exposure to nanoparticles}

In this experiment, we further investigated interference of nanoparticles $\mathrm{ZnO}(0.5 \mathrm{mg} / \mathrm{ml}), \mathrm{TiO}_{2}(0.5 \mathrm{mg} / \mathrm{ml})$, and $\mathrm{SiO}_{2}(1 \mathrm{mg} / \mathrm{ml})$ on quantifications of $S$. enterica Newport, S. epidermidis, E. faecalis, and E. coli with various bacterial concentrations by comparison of flow cytometry with optical density measurement. As shown in Table 4, with the decreasing concentrations of the tested bacteria, no apparent interference of the nanoparticles on quantifications of all four bacterial species by flow cytometry was observed. However, with decreasing concentrations of the tested bacteria, the adverse effect on quantification of bacteria was dramatically increased using the spectrophotometer method of optical density measurement, which reflected a progressively worse estimate of the bacterial counts as the ratio of numbers of bacteria and nanoparticles in the suspension decreased. For example, in the presence of $0.1 \mathrm{mg} \mathrm{TiO}_{2}$, number of S. enterica Newport cells could not be detected due to high background interference from the nanoparticles in the samples. S. enterica Newport, S. epidermidis, E. faecalis could not be quantified in the presence of $0.5 \mathrm{mg} / \mathrm{ml} \mathrm{TiO}_{2}$. The data obtained from the bacterial quantification in the presence of $0.5 \mathrm{mg} / \mathrm{ml}$ of $\mathrm{ZnO}$ were either not able to be detected or not accurate. Due to lower interference of $\mathrm{SiO}_{2}$ at $1 \mathrm{mg} / \mathrm{ml}$ on the bacterial quantification, there was no apparent difference between flow cytometry and optical density measurement (Table 4).

\section{Conclusions}

In summary, this study compared three most commonly used bacterial quantification methods including colony counts, spectrophotometer method of optical density measurement, and flow cytometry in the presence of metal oxide nanoparticles. Our results demonstrated that flow cytometry is the best method with no apparent interference by the nanoparticles, indicating that it is suitable for rapid, accurate and automatic detection of bacteria. Flow cytometry is also able to detect both live and dead bacterial cells and allows detection of all bacteria including those that are uncultured. Although the bacterial quantification determined by plate counts was not affected by the nanoparticles, it was time consuming, less accurate and not suitable for automation. The spectrophotometer method using optical density measurement was the most unreliable method to quantify and detect bacteria in the presence of oxide nanoparticles. The data presented in this study indicated that flow cytometry method for bacterial quantification is superior to the other two methods. This study provides data examining the potential interference of oxide nanoparticles on bacterial quantification. The information provided here will be useful in the assessment of bacterial contamination in food, drug and cosmetic products containing nanoparticles. Future studies on other nanoparticles and limit of the bacterial detection by FMC are warranted.

\section{Methods}

Materials and preparation of nanoparticle suspensions $\mathrm{ZnO}$ (purity >97\%), $\mathrm{TiO}_{2}$ (purity $\geq 99.5 \%$ ), and $\mathrm{SiO}_{2}$ (purity $99.5 \%$ ) nanoparticles were purchased from Sigma Chemical Co. The LIVE/DEAD BacLight bacterial viability and counting kit containing solutions of $3.34 \mathrm{mM}$ SYTO9 in dimethyl sulfoside (DMSO, $200 \mu \mathrm{l}$ ), $20 \mathrm{mM}$ propidium iodide (PI) in DMSO $(200 \mu \mathrm{l})$ and a calibrated suspension of microspheres (diameter: $6 \mu \mathrm{m}, 1 \mathrm{ml}$; concentration: $1.0 \times 10^{8}$ beads $/ \mathrm{ml}$ ) and SYTO 9 green fluorescent nucleic acid stain ( $5 \mathrm{mM}$ solution in DMSO, $100 \mu \mathrm{l}$ ) were purchased from Molecular Probes (Eugene, Oregon). Suspensions of the nanoparticles were prepared with Milli-Q water by means of ultrasonic vibration in a BRANSON 3200 UltraSonic Cleaner for $30 \mathrm{~min}$ and the stock solutions were vortexed briefly before each use [40-42].

\section{Physical and chemical characterizations of nanomaterials}

The size, shape and morphology of $\mathrm{ZnO}, \mathrm{TiO}_{2}$ or $\mathrm{SiO}_{2}$ nanoparticles were determined using transmission electron microscopy (TEM). The nanoparticles were homogeneously 
Table 3 Interference of oxide nanoparticles on bacterial quantification

\begin{tabular}{|c|c|c|c|c|c|c|c|c|c|c|c|c|}
\hline \multirow{4}{*}{$\begin{array}{l}\text { Nanoparticles } \\
\text { (mg/ml) }\end{array}$} & \multicolumn{4}{|l|}{$\mathrm{ZnO}$} & \multicolumn{4}{|l|}{$\mathrm{TiO}_{2}$} & \multicolumn{4}{|l|}{$\mathrm{SiO}_{2}$} \\
\hline & \multicolumn{4}{|c|}{ S. enterica Newport (cells/ml) ${ }^{a}$} & \multicolumn{4}{|c|}{ S. enterica Newport (cells/ml) } & \multicolumn{4}{|c|}{ S. enterica Newport (cells/ml) } \\
\hline & \multicolumn{2}{|l|}{ FMC } & \multirow[t]{2}{*}{ CFU } & \multirow[t]{2}{*}{$\mathrm{OD}_{660} \mathrm{~b}$} & \multicolumn{2}{|l|}{ FMC } & \multirow[t]{2}{*}{ CFU } & \multirow[t]{2}{*}{$\mathrm{OD}_{660}$} & \multicolumn{2}{|l|}{ FMC } & \multirow[t]{2}{*}{ CFU } & \multirow[t]{2}{*}{$\mathrm{OD}_{660}$} \\
\hline & Total & Live & & & Total & Live & & & Total & Live & & \\
\hline 0 & $1.37 \times 10^{9}$ & $1.36 \times 10^{9}$ & $8.17 \times 10^{8}$ & $1.37 \times 10^{9}$ & $1.23 \times 10^{9}$ & $1.22 \times 10^{9}$ & $1.18 \times 10^{9}$ & $1.23 \times 10^{9}$ & $1.28 \times 10^{9}$ & $1.26 \times 10^{9}$ & $6.32 \times 10^{8}$ & $1.28 \times 10^{9}$ \\
\hline 0.1 & $1.31 \times 10^{9}$ & $1.30 \times 10^{9}$ & $1.00 \times 10^{9}$ & $1.46 \times 10^{9}$ & $1.00 \times 10^{9}$ & $9.94 \times 10^{8}$ & $7.00 \times 10^{8}$ & $9.16 \times 10^{8}$ & $1.23 \times 10^{9}$ & $1.22 \times 10^{9}$ & $6.50 \times 10^{8}$ & $1.20 \times 10^{9}$ \\
\hline 0.2 & $1.29 \times 10^{9}$ & $1.28 \times 10^{9}$ & $5.83 \times 10^{8}$ & $1.28 \times 10^{9}$ & $8.15 \times 10^{8}$ & $8.05 \times 10^{8}$ & $5.67 \times 10^{8}$ & $5.89 \times 10^{8}$ & $1.22 \times 10^{9}$ & $1.20 \times 10^{9}$ & $5.83 \times 10^{8}$ & $1.18 \times 10^{9}$ \\
\hline 0.3 & $1.27 \times 10^{9}$ & $1.14 \times 10^{9}$ & $7.00 \times 10^{8}$ & $1.19 \times 10^{9}$ & $7.14 \times 10^{8}$ & $7.06 \times 10^{8}$ & $5.50 \times 10^{8}$ & $3.23 \times 10^{8}$ & $1.20 \times 10^{9}$ & $1.18 \times 10^{9}$ & $5.83 \times 10^{8}$ & $1.16 \times 10^{9}$ \\
\hline 0.5 & $1.23 \times 10^{9}$ & $1.21 \times 10^{9}$ & $6.33 \times 10^{8}$ & $1.01 \times 10^{9}$ & $4.26 \times 10^{8}$ & $4.13 \times 10^{8}$ & $4.33 \times 10^{8}$ & - & $1.24 \times 10^{9}$ & $1.21 \times 10^{9}$ & $5.67 \times 10^{8}$ & $1.15 \times 10^{9}$ \\
\hline \multirow[t]{2}{*}{1} & $1.12 \times 10^{9}$ & $1.10 \times 10^{9}$ & $5.00 \times 10^{8}$ & $7.15 \times 10^{8}$ & $2.41 \times 10^{8}$ & $2.35 \times 10^{8}$ & $1.50 \times 10^{8}$ & - & $1.22 \times 10^{9}$ & $1.20 \times 10^{9}$ & $7.17 \times 10^{8}$ & $1.09 \times 10^{9}$ \\
\hline & \multicolumn{4}{|c|}{ S. epidermidis ATCC 12228 (cells/ml) } & \multicolumn{4}{|c|}{ S. epidermidis ATCC 12228 (cells/ml) } & \multicolumn{4}{|c|}{ S. epidermidis ATCC 12228 (cells/ml) } \\
\hline 0 & $3.53 \times 10^{8}$ & $3.46 \times 10^{8}$ & $9.33 \times 10^{7}$ & $3.53 \times 10^{8}$ & $4.46 \times 10^{8}$ & $4.40 \times 10^{8}$ & $1.20 \times 10^{8}$ & $4.46 \times 10^{8}$ & $5.20 \times 10^{8}$ & $4.74 \times 10^{8}$ & $2.00 \times 10^{8}$ & $5.20 \times 10^{8}$ \\
\hline 0.1 & $2.13 \times 10^{8}$ & $1.94 \times 10^{8}$ & $2.18 \times 10^{7}$ & $2.73 \times 10^{8}$ & $1.21 \times 10^{8}$ & $1.19 \times 10^{8}$ & $2.00 \times 10^{7}$ & - & $1.06 \times 10^{8}$ & $9.57 \times 10^{7}$ & $1.18 \times 10^{8}$ & $4.48 \times 10^{8}$ \\
\hline 0.2 & $1.37 \times 10^{8}$ & $1.18 \times 10^{8}$ & $1.63 \times 10^{7}$ & $1.23 \times 10^{8}$ & $2.65 \times 10^{7}$ & $2.62 \times 10^{7}$ & $2.00 \times 10^{7}$ & - & $7.27 \times 10^{7}$ & $6.55 \times 10^{7}$ & $6.50 \times 10^{7}$ & $4.54 \times 10^{8}$ \\
\hline 0.3 & $1.71 \times 10^{7}$ & $1.45 \times 10^{7}$ & $1.37 \times 10^{7}$ & $3.20 \times 10^{8}$ & $1.46 \times 10^{7}$ & $1.44 \times 10^{7}$ & $3.33 \times 10^{7}$ & - & $5.13 \times 10^{7}$ & $4.60 \times 10^{7}$ & $5.00 \times 10^{7}$ & $5.00 \times 10^{8}$ \\
\hline 0.5 & $1.65 \times 10^{7}$ & $1.45 \times 10^{7}$ & $1.33 \times 10^{7}$ & $1.85 \times 10^{8}$ & $6.47 \times 10^{6}$ & $6.40 \times 10^{6}$ & $5.83 \times 10^{7}$ & - & $6.72 \times 10^{7}$ & $6.32 \times 10^{7}$ & $5.83 \times 10^{7}$ & $4.75 \times 10^{8}$ \\
\hline \multirow[t]{2}{*}{1} & $3.31 \times 10^{7}$ & $3.00 \times 10^{7}$ & $1.10 \times 10^{7}$ & - & $6.20 \times 10^{7}$ & $6.11 \times 10^{7}$ & $1.07 \times 10^{8}$ & - & $2.21 \times 10^{8}$ & $2.04 \times 10^{8}$ & $1.18 \times 10^{8}$ & $4.84 \times 10^{8}$ \\
\hline & \multicolumn{4}{|c|}{ E. faecalis ATCC 27274 (cells/ml) } & \multicolumn{4}{|c|}{ E. faecalis ATCC 27274 (cells/ml) } & \multicolumn{4}{|c|}{ E. faecalis ATCC 27274 (cells/ml) } \\
\hline 0 & $2.29 \times 10^{9}$ & $2.28 \times 10^{9}$ & $1.17 \times 10^{9}$ & $2.29 \times 10^{9}$ & $2.21 \times 10^{9}$ & $2.17 \times 10^{9}$ & $1.07 \times 10^{9}$ & $2.21 \times 10^{9}$ & $2.47 \times 10^{9}$ & $2.42 \times 10^{9}$ & $1.87 \times 10^{9}$ & $2.47 \times 10^{9}$ \\
\hline 0.1 & $2.14 \times 10^{9}$ & $2.06 \times 10^{9}$ & $1.50 \times 10^{9}$ & $2.13 \times 10^{9}$ & $1.84 \times 10^{9}$ & $1.81 \times 10^{9}$ & $9.50 \times 10^{8}$ & $1.69 \times 10^{9}$ & $2.17 \times 10^{9}$ & $2.11 \times 10^{9}$ & $1.22 \times 10^{9}$ & $2.40 \times 10^{9}$ \\
\hline 0.2 & $2.0 \times 10^{9}$ & $1.92 \times 10^{9}$ & $1.42 \times 10^{9}$ & $1.73 \times 10^{9}$ & $1.42 \times 10^{9}$ & $1.40 \times 10^{9}$ & $5.50 \times 10^{8}$ & $1.07 \times 10^{9}$ & $1.64 \times 10^{9}$ & $1.61 \times 10^{9}$ & $1.18 \times 10^{9}$ & $2.30 \times 10^{9}$ \\
\hline 0.3 & $1.47 \times 10^{9}$ & $1.44 \times 10^{9}$ & $1.28 \times 10^{9}$ & $1.54 \times 10^{9}$ & $1.23 \times 10^{9}$ & $1.22 \times 10^{9}$ & $3.80 \times 10^{8}$ & $5.26 \times 10^{8}$ & $1.34 \times 10^{9}$ & $1.33 \times 10^{9}$ & $1.14 \times 10^{9}$ & $2.23 \times 10^{9}$ \\
\hline 0.5 & $1.45 \times 10^{9}$ & $1.40 \times 10^{9}$ & $1.15 \times 10^{9}$ & $8.57 \times 10^{8}$ & $5.58 \times 10^{8}$ & $5.54 \times 10^{8}$ & $1.30 \times 10^{8}$ & - & $8.69 \times 10^{8}$ & $8.59 \times 10^{8}$ & $7.00 \times 10^{8}$ & $2.10 \times 10^{9}$ \\
\hline \multirow[t]{2}{*}{1} & $1.07 \times 10^{9}$ & $1.03 \times 10^{9}$ & $7.00 \times 10^{8}$ & - & $1.70 \times 10^{6}$ & $1.60 \times 10^{6}$ & $2.95 \times 10^{6}$ & - & $4.44 \times 10^{8}$ & $4.33 \times 10^{8}$ & $5.00 \times 10^{8}$ & $1.90 \times 10^{9}$ \\
\hline & \multicolumn{4}{|c|}{ E. coli ATCC 25922 (cells/ml) } & \multicolumn{4}{|c|}{ E. coli ATCC 25922 (cells/ml) } & E. coli ATC & 22 (cells $/ \mathrm{m}$ & & \\
\hline 0 & $6.56 \times 10^{8}$ & $5.64 \times 10^{8}$ & $3.98 \times 10^{8}$ & $6.65 \times 10^{8}$ & $6.41 \times 10^{8}$ & $6.32 \times 10^{8}$ & $6.83 \times 10^{8}$ & $6.41 \times 10^{8}$ & $5.52 \times 10^{8}$ & $5.46 \times 10^{8}$ & $5.67 \times 10^{8}$ & $5.52 \times 10^{8}$ \\
\hline 0.1 & $5.22 \times 10^{8}$ & $4.95 \times 10^{8}$ & $3.93 \times 10^{8}$ & $6.18 \times 10^{8}$ & $3.28 \times 10^{8}$ & $3.26 \times 10^{8}$ & $8.33 \times 10^{7}$ & $4.86 \times 10^{8}$ & $3.73 \times 10^{8}$ & $3.68 \times 10^{8}$ & $2.83 \times 10^{8}$ & $5.21 \times 10^{8}$ \\
\hline 0.2 & $4.50 \times 10^{8}$ & $4.17 \times 10^{8}$ & $3.88 \times 10^{8}$ & $5.56 \times 10^{8}$ & $7.67 \times 10^{7}$ & $7.61 \times 10^{7}$ & $1.17 \times 10^{7}$ & $3.07 \times 10^{8}$ & $2.52 \times 10^{8}$ & $2.49 \times 10^{8}$ & $2.17 \times 10^{8}$ & $5.08 \times 10^{8}$ \\
\hline 0.3 & $3.65 \times 10^{8}$ & $3.54 \times 10^{8}$ & $3.87 \times 10^{8}$ & $4.97 \times 10^{8}$ & $1.90 \times 10^{7}$ & $1.88 \times 10^{7}$ & $1.17 \times 10^{7}$ & $1.63 \times 10^{8}$ & $2.19 \times 10^{8}$ & $2.16 \times 10^{8}$ & $1.50 \times 10^{8}$ & $5.11 \times 10^{8}$ \\
\hline 0.5 & $1.36 \times 10^{8}$ & $1.17 \times 10^{8}$ & $2.93 \times 10^{8}$ & $2.89 \times 10^{8}$ & $7.13 \times 10^{6}$ & $6.97 \times 10^{6}$ & $9.02 \times 10^{6}$ & - & $2.03 \times 10^{8}$ & $2.02 \times 10^{8}$ & $2.50 \times 10^{8}$ & $4.76 \times 10^{8}$ \\
\hline 1 & $1.43 \times 10^{8}$ & $1.37 \times 10^{8}$ & $3.10 \times 10^{8}$ & $1.59 \times 10^{8}$ & $2.21 \times 10^{7}$ & $2.18 \times 10^{7}$ & $4.58 \times 10^{7}$ & - & $2.38 \times 10^{8}$ & $2.37 \times 10^{8}$ & $2.83 \times 10^{8}$ & $4.67 \times 10^{8}$ \\
\hline
\end{tabular}

${ }^{a}$ Bacterial cell concentrations were measured by flow cytometry (FCM), culture-based counting for colony-forming units (CFU), and spectrophotometer method of optical density (OD) measurement after 1 hr exposure to different concentrations of $\mathrm{ZnO}, \mathrm{TiO}_{2}$ and $\mathrm{SiO}_{2}$ nanoparticles; inoculum used for each experiment was indicated in the control samples, i.e. no nanoparticles.

${ }^{b}$ Presented data were converted from each sample cell concentration according to the each species standard curve of cell/ml vs $\mathrm{OD}_{660}$ and as mean of triplicate with standard deviations (SD) of $<5 \%$ from $\mathrm{FCM}$ and $\mathrm{OD} \mathrm{D}^{600}$ and $<10 \%$ from CFU. Inoculum used for each experiment was indicated in the control samples, i.e. no nanoparticles. 

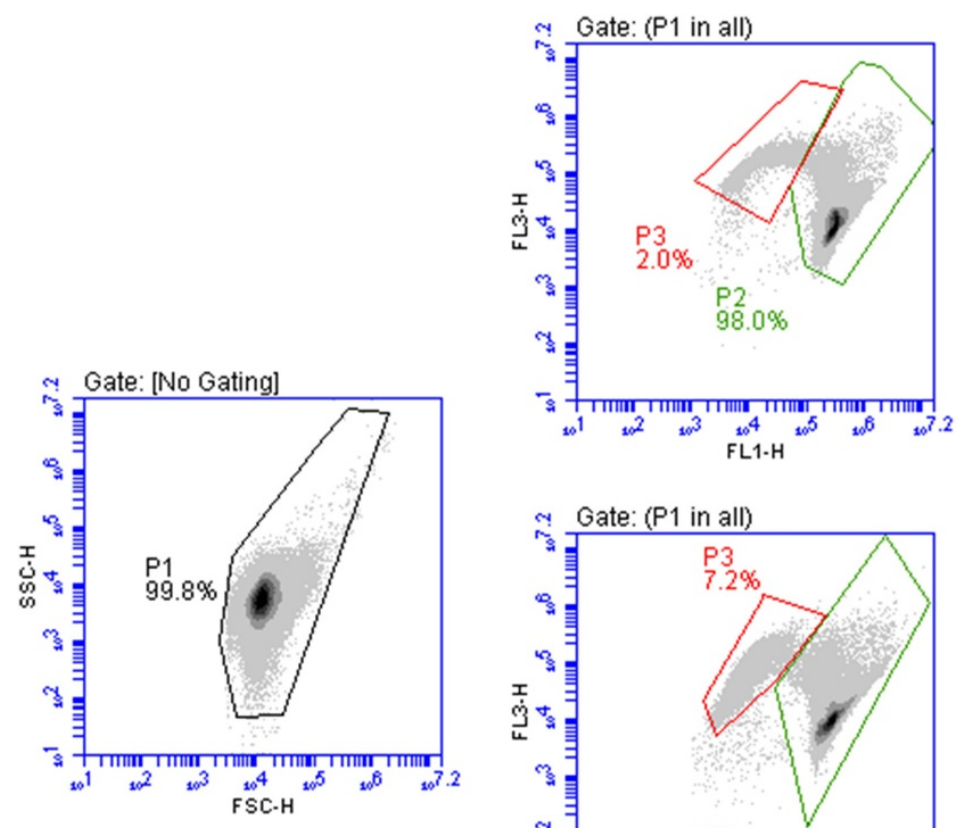

\section{Control}

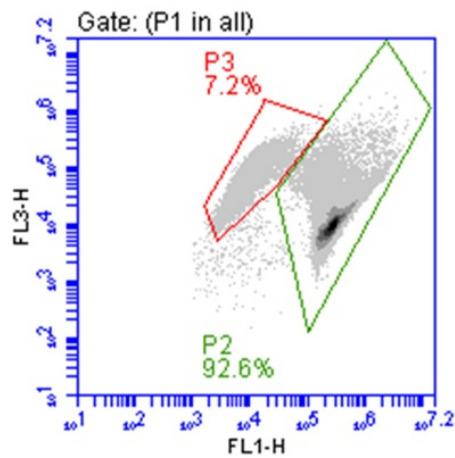

\section{$\mathrm{ZnO}$ \\ (0.2 mg/ml)}

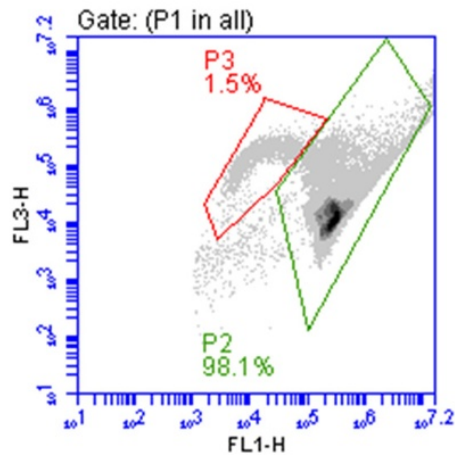

$\mathrm{TiO}_{2}$

(0.2 mg/ml)

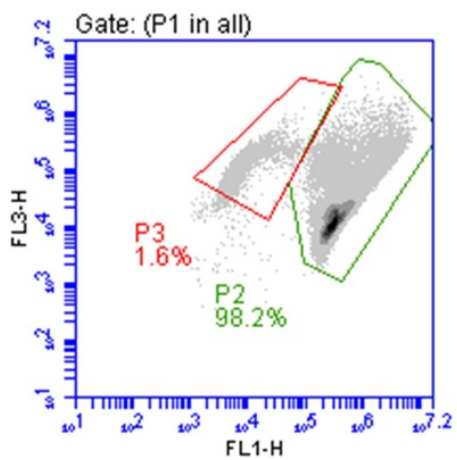

$\mathrm{SiO}_{2}$

(0.2 mg/ml)

Figure 2 Examples of flow cytometric for $\mathrm{E}$. faecalis exposure to nanoparticles- $\mathrm{ZnO}, \mathrm{TiO}_{2}$, and $\mathrm{SiO}_{2}$ at concentration of $0.2 \mathrm{mg} / \mathrm{ml}$.

Fluorescence (FL1-H/FL3-H) was tested from bacterial cells inside gate P1 in a FSC-H (forward scatter-H)/SSC-H (side scatter-H) density plots. Live bacterial cells (gate P2); dead bacterial cells (gate P3). 
Table 4 Quantification of bacterial cells at various concentrations in the presence of oxide nanoparticles

\begin{tabular}{|c|c|c|c|c|c|c|c|c|c|c|c|c|}
\hline \multirow[t]{3}{*}{ Strain name } & \multicolumn{3}{|c|}{ Control (No nanoparticles) $^{a}$} & \multicolumn{3}{|c|}{$\mathrm{ZnO}(0.5 \mathrm{mg} / \mathrm{ml})$} & \multicolumn{3}{|c|}{$\mathrm{TiO}_{2}(0.5 \mathrm{mg} / \mathrm{ml})$} & \multicolumn{3}{|l|}{$\mathrm{SiO}_{2}(1 \mathrm{mg} / \mathrm{ml})$} \\
\hline & \multicolumn{2}{|l|}{ FCM } & \multirow[t]{2}{*}{$\mathrm{OD}_{660}{ }^{\mathrm{b}}$} & \multicolumn{2}{|l|}{ FCM } & \multirow[t]{2}{*}{$\mathrm{OD}_{660}$} & \multicolumn{2}{|l|}{ FCM } & \multirow[t]{2}{*}{$\mathrm{OD}_{660}$} & \multicolumn{2}{|l|}{ FCM } & \multirow[t]{2}{*}{$\mathrm{OD}_{660}$} \\
\hline & Total cell no. & Live cell no. & & Total cell no. & Live cell no. & & Total cell no. & Live cell no. & & Total cell no. & Live cell no. & \\
\hline \multirow[t]{5}{*}{ S. enterica Newport } & $1.34 \times 10^{9}$ & $1.31 \times 10^{9}$ & $1.34 \times 10^{9}$ & $1.20 \times 10^{9}$ & $1.17 \times 10^{9}$ & $7.47 \times 10^{8}$ & $4.72 \times 10^{8}$ & $4.63 \times 10^{8}$ & - & $1.29 \times 10^{9}$ & $1.29 \times 10^{9}$ & $1.36 \times 10^{9}$ \\
\hline & $6.76 \times 10^{8}$ & $6.61 \times 10^{8}$ & $7.45 \times 10^{8}$ & $5.18 \times 10^{8}$ & $5.06 \times 10^{8}$ & $1.73 \times 10^{8}$ & $9.58 \times 10^{7}$ & $9.21 \times 10^{7}$ & - & $6.07 \times 10^{8}$ & $6.06 \times 10^{8}$ & $7.91 \times 10^{7}$ \\
\hline & $3.30 \times 10^{8}$ & $3.20 \times 10^{8}$ & $3.79 \times 10^{8}$ & $2.19 \times 10^{8}$ & $2.13 \times 10^{8}$ & $-c$ & $7.78 \times 10^{7}$ & $7.34 \times 10^{7}$ & - & $3.04 \times 10^{8}$ & $3.03 \times 10^{8}$ & $4.47 \times 10^{8}$ \\
\hline & $1.51 \times 10^{8}$ & $1.47 \times 10^{8}$ & $1.96 \times 10^{8}$ & $8.89 \times 10^{7}$ & $8.77 \times 10^{7}$ & - & $6.56 \times 10^{7}$ & $6.21 \times 10^{7}$ & - & $1.19 \times 10^{8}$ & $1.18 \times 10^{8}$ & $2.87 \times 10^{8}$ \\
\hline & $1.18 \times 10^{8}$ & $1.14 \times 10^{8}$ & $1.50 \times 10^{8}$ & $7.51 \times 10^{7}$ & $7.37 \times 10^{7}$ & - & $6.01 \times 10^{7}$ & $5.68 \times 10^{7}$ & - & $1.00 \times 10^{8}$ & $9.99 \times 10^{7}$ & $1.73 \times 10^{8}$ \\
\hline \multirow[t]{5}{*}{ S. epidermidis } & $3.43 \times 10^{8}$ & $3.38 \times 10^{8}$ & $3.43 \times 10^{8}$ & $1.65 \times 10^{7}$ & $1.50 \times 10^{7}$ & $1.59 \times 10^{8}$ & $3.06 \times 10^{7}$ & $3.03 \times 10^{7}$ & - & $1.75 \times 10^{8}$ & $1.73 \times 10^{8}$ & $3.96 \times 10^{8}$ \\
\hline & $1.73 \times 10^{8}$ & $1.70 \times 10^{8}$ & $1.59 \times 10^{8}$ & $4.37 \times 10^{7}$ & $3.66 \times 10^{7}$ & $1.19 \times 10^{8}$ & $6.91 \times 10^{7}$ & $6.89 \times 10^{7}$ & - & $1.57 \times 10^{8}$ & $1.55 \times 10^{8}$ & $1.59 \times 10^{8}$ \\
\hline & $8.41 \times 10^{7}$ & $2.96 \times 10^{7}$ & $6.67 \times 10^{7}$ & $3.67 \times 10^{7}$ & $2.94 \times 10^{7}$ & $5.32 \times 10^{7}$ & $5.34 \times 10^{7}$ & $5.30 \times 10^{7}$ & - & $7.56 \times 10^{7}$ & $7.42 \times 10^{7}$ & $7.96 \times 10^{7}$ \\
\hline & $4.10 \times 10^{7}$ & $1.87 \times 10^{7}$ & $2.69 \times 10^{7}$ & $2.14 \times 10^{7}$ & $1.63 \times 10^{7}$ & $3.98 \times 10^{7}$ & $2.88 \times 10^{7}$ & $2.85 \times 10^{7}$ & - & $3.57 \times 10^{7}$ & $3.48 \times 10^{7}$ & $2.69 \times 10^{7}$ \\
\hline & $4.04 \times 10^{7}$ & $1.48 \times 10^{7}$ & $1.37 \times 10^{7}$ & $1.74 \times 10^{7}$ & $1.32 \times 10^{7}$ & $2.69 \times 10^{7}$ & $3.27 \times 10^{7}$ & $3.25 \times 10^{7}$ & 0 & $3.99 \times 10^{7}$ & $3.87 \times 10^{7}$ & $2.69 \times 10^{7}$ \\
\hline \multirow[t]{5}{*}{ E. faecalis } & $2.33 \times 10^{9}$ & $2.32 \times 10^{9}$ & $2.33 \times 10^{9}$ & $1.20 \times 10^{9}$ & $1.16 \times 10^{9}$ & $8.82 \times 10^{8}$ & $5.54 \times 10^{8}$ & $5.33 \times 10^{8}$ & - & $7.10 \times 10^{8}$ & $7.07 \times 10^{8}$ & $2.02 \times 10^{9}$ \\
\hline & $1.20 \times 10^{9}$ & $1.19 \times 10^{9}$ & $1.27 \times 10^{9}$ & $1.44 \times 10^{8}$ & $1.26 \times 10^{8}$ & - & $8.43 \times 10^{6}$ & $8.10 \times 10^{6}$ & - & $3.17 \times 10^{8}$ & $3.14 \times 10^{8}$ & $1.41 \times 10^{9}$ \\
\hline & $5.86 \times 10^{8}$ & $5.68 \times 10^{8}$ & $5.94 \times 10^{8}$ & $4.26 \times 10^{7}$ & $4.00 \times 10^{7}$ & - & $4.30 \times 10^{6}$ & $4.20 \times 10^{6}$ & - & $1.43 \times 10^{8}$ & $1.42 \times 10^{8}$ & $5.94 \times 10^{8}$ \\
\hline & $2.78 \times 10^{8}$ & $2.74 \times 10^{8}$ & $2.60 \times 10^{8}$ & $4.00 \times 10^{7}$ & $3.87 \times 10^{7}$ & - & $2.02 \times 10^{7}$ & $1.98 \times 10^{7}$ & - & $1.20 \times 10^{8}$ & $1.17 \times 10^{8}$ & $3.37 \times 10^{8}$ \\
\hline & $2.27 \times 10^{8}$ & $2.21 \times 10^{8}$ & $2.08 \times 10^{8}$ & $3.62 \times 10^{7}$ & $3.53 \times 10^{7}$ & - & $2.52 \times 10^{7}$ & $2.48 \times 10^{7}$ & - & $1.16 \times 10^{8}$ & $1.13 \times 10^{8}$ & $2.86 \times 10^{8}$ \\
\hline \multirow[t]{5}{*}{ E. coli } & $6.04 \times 10^{8}$ & $5.57 \times 10^{8}$ & $6.04 \times 10^{8}$ & $8.96 \times 10^{7}$ & $7.17 \times 10^{7}$ & $2.94 \times 10^{8}$ & $1.69 \times 10^{7}$ & $1.50 \times 10^{7}$ & - & $2.17 \times 10^{8}$ & $2.04 \times 10^{8}$ & $5.51 \times 10^{8}$ \\
\hline & $2.98 \times 10^{8}$ & $2.76 \times 10^{8}$ & $3.21 \times 10^{8}$ & $6.04 \times 10^{7}$ & $4.17 \times 10^{7}$ & $9.85 \times 10^{7}$ & $4.89 \times 10^{7}$ & $4.39 \times 10^{7}$ & - & $2.07 \times 10^{8}$ & $1.93 \times 10^{8}$ & $3.38 \times 10^{8}$ \\
\hline & $1.51 \times 10^{8}$ & $1.41 \times 10^{8}$ & $1.52 \times 10^{8}$ & $4.80 \times 10^{7}$ & $3.42 \times 10^{7}$ & - & $5.99 \times 10^{7}$ & $5.11 \times 10^{7}$ & - & $1.38 \times 10^{8}$ & $1.23 \times 10^{8}$ & $1.87 \times 10^{8}$ \\
\hline & $6.55 \times 10^{7}$ & $6.02 \times 10^{7}$ & $6.34 \times 10^{7}$ & $3.75 \times 10^{7}$ & $2.51 \times 10^{7}$ & - & $5.12 \times 10^{7}$ & $4.20 \times 10^{7}$ & - & $6.31 \times 10^{7}$ & $5.55 \times 10^{7}$ & $8.11 \times 10^{7}$ \\
\hline & $5.47 \times 10^{7}$ & $5.20 \times 10^{7}$ & $3.68 \times 10^{7}$ & $3.28 \times 10^{7}$ & $1.87 \times 10^{7}$ & - & $4.47 \times 10^{7}$ & $4.07 \times 10^{7}$ & - & $5.10 \times 10^{7}$ & $4.44 \times 10^{7}$ & $8.11 \times 10^{7}$ \\
\hline
\end{tabular}

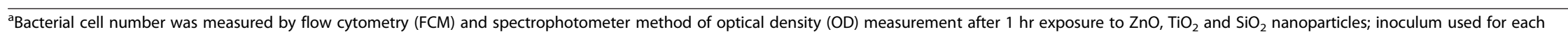
experiment was indicated in the control samples, i.e. no nanoparticles.

${ }^{b}$ Presented data were converted from each sample cells concentration according to the each species standard curve of cell $/ \mathrm{ml}$ vs $\mathrm{OD}_{660}$ and as mean of triplicate with standard deviations (SD) of $<5 \%$. 'Value was negative. 
dispersed in Milli-Q water, and $3 \mu \mathrm{L}$ suspensions was deposited on the TEM grid, dried, and evacuated before analysis. Images were collected using a field emission JEM-2100 F (JEOL, Tokyo, Japan) equipped with a CCD camera in high resolution mode with an acceleration voltage of $100 \mathrm{kV}$.

The hydrodynamic size and zeta potential were measured in Milli-Q water using a Zetasizer (Malvern, Worcestershire, UK) as described in previous study [43]. Briefly, the nanoparticle samples were measured after dilution of a nanoparticle stock solution to $50 \mu \mathrm{g} / \mathrm{ml}$ in Milli-Q water. These dilutions were sonicated for $30 \mathrm{~min}$ and vortexed briefly to provide a homogenous dispersion. For the size measurement, $70 \mu \mathrm{L}$ of the diluted dispersion nanoparticles was transferred to a cuvette for dynamic size measurement; for zeta potential measurement, a Malvern zeta potential cell was washed three times with ultrapure water followed by transferring $850 \mu \mathrm{l}$ of diluted dispersion nanoparticles to this cell to measure the zeta potential. The concentration of the samples and experimental methods were optimized to assure the quality of the data. NIST standard gold nanoparticles $(10 \mathrm{~nm}, 30 \mathrm{~nm}$, and $60 \mathrm{~nm}$ ) were used in the validation of the instrument. Both size and zeta potential were measured at least three times. The data were calculated as the average size or zeta potential of nanoparticles.

\section{Bacterial strains and culture conditions}

Four bacterial species were chosen for all experiments (Table 2). The bacterial stock cultures were stored in freezer $\left(-80^{\circ} \mathrm{C}\right)$ with glycerol to a final concentration of 15\%. E. faecalis and E. coli from the glycerol stocks were streaked into brain heart infusion (BHI) agar plates at $37^{\circ} \mathrm{C}$ overnight in an anaerobic chamber (Coy Laboratory Products INC.). For S. enterica Newport and S. epidermidis, the plates were grown under aerobic condition. One colony was picked by a loop and inoculated into a 50-ml Falcon centrifuge tube containing $10 \mathrm{ml} \mathrm{BHI}$ medium. The cultures were incubated anaerobically or aerobically in static conditions at $37^{\circ} \mathrm{C}$ overnight for use as seed cultures. Each seed culture of the bacteria was inoculated into BHI medium with an inoculation ratio of $1 \%$ (v/v), except S. epidermidis with $10 \%(\mathrm{v} / \mathrm{v})$ of inoculation into $\mathrm{BHI}$ medium, and then the cultures were incubated at $37^{\circ} \mathrm{C}$ under anaerobic or aerobic condition without agitation for 4-6 hrs to enter the exponential phase based on our preliminary experiments [40]. The cells were collected by centrifugation $\left(10,000 \mathrm{rpm}, 2 \mathrm{~min}, 4^{\circ} \mathrm{C}\right)$, washed twice and then re-suspended in sterile saline solution $(0.85 \%$ $\mathrm{NaCl}$ ), which served as experimental bacterial cells. To measure the bacterial numbers in the presence of different concentrations of nano $\mathrm{ZnO}, \mathrm{TiO}_{2}$, and $\mathrm{SiO}_{2}$, various concentrations of the nanoparticles (final concentrations were $0,0.1,0.2,0.3,0.5,1 \mathrm{mg} / \mathrm{ml}$ ) based on our based on our preliminary experiments were added into each bacterial cell re-suspension and mixed well by vortexing, leaving one as a control without nanoparticles, but same volume of Milli-Q water, and then kept in the dark for $1 \mathrm{hr}$ at $4^{\circ} \mathrm{C}$ [39]. In order to test the different bacterial concentrations after exposure to the nanoparticles, the initial bacterial resuspension with approximately $10^{8}-10^{9}$ cells $/ \mathrm{ml}$ was serially diluted $(0,-2,-4,-8,-10$ fold $)$ in saline solution and then mixed well with each nanoparticles at final concentration of $0.5 \mathrm{mg} / \mathrm{ml}, 0.5 \mathrm{mg} / \mathrm{ml}$ and $1 \mathrm{mg} / \mathrm{ml}$ for $\mathrm{ZnO}$, $\mathrm{TiO}_{2}$ and $\mathrm{SiO}_{2}$, respectively. All the samples were kept under the same conditions as mentioned above. A control (containing saline solution and nanoparticles without bacterial cells) was included in all experiments and kept under same conditions. All experiments were carried out in triplicates. After $1 \mathrm{hr}$ exposure to the nanoparticles, the bacterial cell concentrations were measured by different methods as mentioned below $[40,41,44,45]$.

\section{Plate counting cell numbers}

Samples were withdrawn and then serially diluted in saline solution. Aliquots of $10 \mu \mathrm{l}$ were spread on BHI-plates. After overnight incubation at $37^{\circ} \mathrm{C}$, colonies on the plates were counted to determine the number of CFU [46].

\section{Optical density measurement}

Aliquots of $200 \mu \mathrm{l}$ were withdrawn, added to a 96-well plate (Corning incorporated, flat bottom, non-lid) and immediately assayed by measuring the optical density in a SpectraMax M2 plate reader (Molecular Devices) at $660 \mathrm{~nm}$ [41]. The absorbance values of the controls were subtracted from the experimental values [36].

\section{Flow cytometry analysis of bacterial cell numbers in combination with LIVE/DEAD BacLight bacterial viability and counting kit}

Samples were collected, diluted and stained according to the manufacture's instruction using the BacLight LIVE/ DEAD bacterial viability and counting kit as described briefly here. Each of $1.5 \mu \mathrm{l}$ of $3.34 \mathrm{mM}$ SYTO 9 green fluorescent nucleic acid dye (Component A) and of $20 \mathrm{mM}$ propidium iodide (Component $\mathrm{B}$ ) was added to the flow cytometry tube containing $1 \mathrm{ml}$ sample. Incubate the sample for 15 minutes at room temperature protected from light. The bacterial cell numbers assay was performed on the Accuri C6 flow cytometry. Fluorescence filters and detectors were all standardized with green fluorescence collected in the FL1 channel (530 \pm $15 \mathrm{~nm}$ ) and red fluorescence collected in the FL3 channel (>670 nm). All parameters were collected as logarithmic signals. A similar setup of parameters was used as described previously [40]. Data were analyzed using CFlow Plus software. In density plots of light scatter properties, bacterial cells were gated from irrelevant counts for 

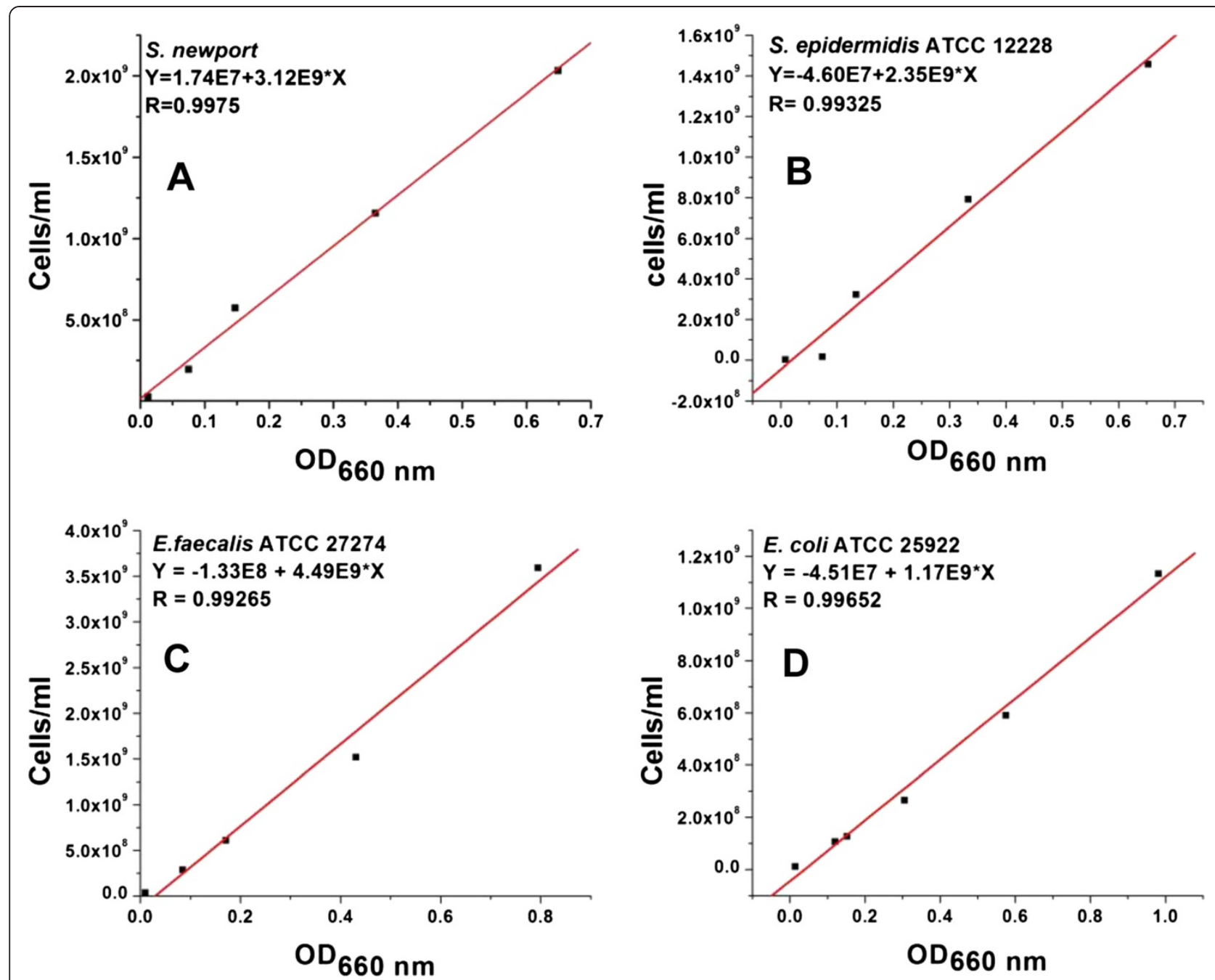

Figure 3 Standard curve of optical density (OD) versus bacterial cell number obtained by flow cytometry (FCM) containing no nanoparticles. A, S. enterica Newport; B, S. epidermidis; C, E. faecalis; D, E. coli. The correlation between OD660 and bacterial cell number for each species was established by means of a standard curve. Data are presented as mean of triplicate with standard deviations (SD) of $<5 \%$. $Y$ is cells $/ \mathrm{ml} ; \mathrm{X}$ is $\mathrm{OD} 660 \mathrm{~nm}$ value; $\mathrm{E}$ is $10 \wedge$; $\mathrm{R}$ is correlation coefficient.

fluorescence analyses. In density plots of fluorescence, the distinct bacterial populations (live cells and damaged or dead cells) were gated based on the different viability stages. Total cell numbers $=$ live cell numbers + dead cell numbers. Accuri C6 flow cytometry was calibrated using 8-peak Spherotech Validation Beads m.

Standard curve of optical density versus cell number for each bacterial stain

Exponentially growing cells of each bacterial species were serially diluted in saline solution in triplicate. Then $\mathrm{OD}_{660}$ of the samples was measured by above mentioned method. Sterile saline solution was used as blanks. For counting cell numbers, the serially diluted bacterial cultures were further diluted to $1 \mathrm{ml}$ with saline solution. Then the total bacterial cell number was analyzed by flow cytometry as mentioned above. The correlation between $\mathrm{OD}_{660}$ and cells number for each bacterial species was established by means of a standard curve (Figure 3).

\section{Competing interests}

The authors declare that they have no competing interests.

Authors' contributions

$H P, Y Z, G H, N K$, and $H C$ performed research and analyzed data. HC conceived and designed the project. HC wrote the paper with help from all authors. The final manuscript was read and approved by all authors.

\section{Acknowledgements}

We would like to thank Drs. Steven L. Foley and Jing Han for their critical review of this manuscript. This study was funded by National Center for Toxicological Research, United States Food and Drug Administration, and supported in part by appointment (H.P.) to the Postgraduate Research Fellowship Program by the Oak Ridge Institute for Science and Education through an interagency agreement between the U.S. Department of Energy and the U.S. Food and Drug Administration. The authors would like to thank 
M. Yvonne Jones for assistance with TEM images. The views presented in this article do not necessarily reflect those of the Food and Drug Administration.

\section{Author details}

'Division of Microbiology, National Center for Toxicological Research, U.S. Food and Drug Administration, Jefferson, AR 72079, USA. ${ }^{2}$ Nanotechnology Core Facility, National Center for Toxicological Research, U.S. Food and Drug Administration, Jefferson, AR 72079, USA. ${ }^{3}$ Department of Clinical Laboratory and Nutritional Sciences, University of Massachusetts Lowell, Lowell, MA 01854, USA.

\section{Received: 17 March 2014 Accepted: 13 August 2014}

Published: 21 August 2014

\section{References}

1. Hajipour MJ, Fromm KM, Ashkarran AA, Jimenez de Aberasturi D, De Larramendi IR, Rojo T, Serpooshan V, Parak WJ, Mahmoudi M: Antibacterial properties of nanoparticles. Trends Biotechnol 2012, 30(10):499-511.

2. Allahverdiyev AM, Abamor ES, Bagirova M, Rafailovich M: Antimicrobial effects of $\mathrm{TiO}(2)$ and $\mathrm{Ag}(2) \mathrm{O}$ nanoparticles against drug-resistant bacteria and leishmania parasites. Future Microbiol 2011, 6(8):933-940.

3. Suresh AK, Pelletier DA, Doktycz MJ: Relating nanomaterial properties and microbial toxicity. Nanoscale 2013, 5(2):463-474.

4. Valdiglesias V, Costa C, Kilic G, Costa S, Pasaro E, Laffon B, Teixeira JP. Neuronal cytotoxicity and genotoxicity induced by zinc oxide nanoparticles. Environ Int 2013, 55:92-100.

5. Warheit DB: How to measure hazards/risks following exposures to nanoscale or pigment-grade titanium dioxide particles. Toxicol Lett 2013, 220(2):193-204.

6. Hoff D, Sheikh L, Bhattacharya S, Nayar S, Webster TJ: Comparison study of ferrofluid and powder iron oxide nanoparticle permeability across the blood-brain barrier. Int J Nanomedicine 2013, 8:703-710.

7. Thorley AJ, Tetley TD: New perspectives in nanomedicine. Pharmacol Ther 2013, 140(2):176-185.

8. Ko H-H, Chen H-T, Yen F-L, Lu W-C, Kuo C-W, Wang M-C: Preparation of $\mathrm{TiO}(2)$ Nanocrystallite Powders Coated with $9 \mathrm{~mol} \% \mathrm{ZnO}$ for Cosmetic Applications in Sunscreens. Int J Mol Sci 2012, 13(2):1658-1669.

9. Battez AH, Gonzalez R, Viesca JL, Fernandez JE, Fernandez JMD, Machado A, Chou R, Riba J: $\mathrm{CuO}, \mathrm{ZrO} 2$ and $\mathrm{ZnO}$ nanoparticles as antiwear additive in oil lubricants. Wear 2008, 265(3-4):422-428.

10. Duncan TV: Applications of nanotechnology in food packaging and food safety: barrier materials, antimicrobials and sensors. J Colloid Interface Sci 2011, 363(1):1-24.

11. Gupta S, Tripathi M: A review of TiO2 nanoparticles. Chin Sci Bull 2011, 56(16):1639-1657.

12. Applerot G, Lipovsky A, Dror R, Perkas N, Nitzan Y, Lubart R, Gedanken A: Enhanced antibacterial activity of nanocrystalline $\mathrm{ZnO}$ Due to increased ROS-mediated cell injury. Adv Funct Mater 2009, 19(6):842-852.

13. Warnes $S L$, Caves V, Keevil CW: Mechanism of copper surface toxicity in Escherichia coli 0157:H7 and Salmonella involves immediate membrane depolarization followed by slower rate of DNA destruction which differs from that observed for Gram-positive bacteria. Environ Microbiol 2012, 14(7):1730-1743

14. Jena P, Mohanty S, Mallick R, Jacob B, Sonawane A: Toxicity and antibacterial assessment of chitosan-coated silver nanoparticles on human pathogens and macrophage cells. Int J Nanomedicine 2012, 7:1805-1818.

15. Wagenvoort JHT, De Brauwer EIGB, Penders RJR, Willems RJ, Top J, Bonten MJ: Environmental survival of vancomycin-resistant Enterococcus faecium. J Hosp Infect 2011, 77(3):282-283.

16. Seil JT, Webster TJ: Antimicrobial applications of nanotechnology: methods and literature. Int J Nanomedicine 2012, 7:2767-2781.

17. Saravanan M, Nanda A: Extracellular synthesis of silver bionanoparticles from Aspergillus clavatus and its antimicrobial activity against MRSA and MRSE. Colloids Surf B: Biointerfaces 2010, 77(2):214-218.

18. De Lima R, Seabra AB, Duran N: Silver nanoparticles: a brief review of cytotoxicity and genotoxicity of chemically and biogenically synthesized nanoparticles. J Appl Toxicol 2012, 32(11):867-879.

19. Bondarenko O, Juganson K, Ivask A, Kasemets K, Mortimer M, Kahru A: Toxicity of $\mathrm{Ag}, \mathrm{CuO}$ and $\mathrm{ZnO}$ nanoparticles to selected environmentally relevant test organisms and mammalian cells in vitro: a critical review. Arch Toxicol 2013, 87(7):1181-1200.

20. Quigley L, O'Sullivan O, Beresford TP, Ross RP, Fitzgerald GF, Cotter PD: Molecular approaches to analysing the microbial composition of raw milk and raw milk cheese. Int J Food Microbiol 2011, 150(2-3):81-94.

21. Fang $H$, Xu J, Ding D, Jackson $S A$, Patel IR, Frye JG, Zou W, Nayak R, Foley $S$, Chen J, Su J, Ye Y, Turner S, Harris S, Zhou G, Cerniglia C, Tong W: An FDA bioinformatics tool for microbial genomics research on molecular characterization of bacterial foodborne pathogens using microarrays. BMC Bioinformatics 2010, 11(Suppl 6):S4.

22. Zhang K, Cheng L, Imazato S, Antonucci JM, Lin NJ, Lin-Gibson S, Bai Y, Xu HHK: Effects of dual antibacterial agents MDPB and nano-silver in primer on microcosm biofilm, cytotoxicity and dentine bond properties. J Dent 2013, 41(5):464-474

23. Koseki S, Nonaka J: Alternative approach to modeling bacterial lag time, using logistic regression as a function of time, temperature, $\mathrm{pH}$, and sodium chloride concentration. Appl Environ Microbiol 2012, 78(17):6103-6112.

24. Schacht VJ, Neumann LV, Sandhi SK, Chen L, Henning T, Klar PJ, Theophel K, Schnell S, Bunge M: Effects of silver nanoparticles on microbial growth dynamics. J Appl Microbiol 2013, 114(1):25-35.

25. Dudak FC, Boyaci IH: Rapid and label-free bacteria detection by surface plasmon resonance (SPR) biosensors. Biotechnol J 2009, 4(7):1003-1011.

26. Vital M, Dignum M, Magic-Knezev A, Ross P, Rietveld L, Hammes F: Flow cytometry and adenosine tri-phosphate analysis: alternative possibilities to evaluate major bacteriological changes in drinking water treatment and distribution systems. Water Res 2012, 46(15):4665-4676.

27. Zahavy E, Ber R, Gur D, Abramovich H, Freeman E, Maoz S, Yitzhaki S: Application of nanoparticles for the detection and sorting of pathogenic bacteria by flow-cytometry. Adv Exp Med Biol 2012, 733:23-36.

28. Masco L, Vanhoutte T, Temmerman R, Swings J, Huys G: Evaluation of real-time PCR targeting the $16 \mathrm{~S}$ rRNA and recA genes for the enumeration of bifidobacteria in probiotic products. Int J Food Microbiol 2007, 113(3):351-357

29. Lazcka O, Del Campo FJ, Munoz FX: Pathogen detection: a perspective of traditional methods and biosensors. Biosens Bioelectron 2007, 22 (7):1205-1217.

30. Davey HM: Life, death, and in-between: meanings and methods in microbiology. Appl Environ Microbiol 2011, 77(16):5571-5576.

31. Kerstens M, Boulet G, Pintelon I, Hellings M, Voeten L, Delputte P, Maes L, $\operatorname{Cos} P$ : Quantification of Candida albicans by flow cytometry using TO-PRO()-3 iodide as a single-stain viability dye. J Microbiol Methods 2013, 92(2):189-191.

32. Lehtinen J, Nuutila J, Lilius E-M: Green fluorescent protein-propidium iodide (GFP-PI) based assay for flow cytometric measurement of bacterial viability. Cytometry A 2004, 60(2):165-172.

33. Hammes F, Egli T: Cytometric methods for measuring bacteria in water: advantages, pitfalls and applications. Anal Bioanal Chem 2010, 397 (3):1083-1095

34. Muller S, Nebe-von-Caron G: Functional single-cell analyses: flow cytometry and cell sorting of microbial populations and communities. FEMS Microbiol Rev 2010, 34(4):554-587.

35. Mallick S, Sharma S, Banerjee M, Ghosh SS, Chattopadhyay A, Paul A: lodine-stabilized Cu nanoparticle chitosan composite for antibacterial applications. ACS Appl Mater Interfaces 2012, 4(3):1313-1323.

36. Sadiq IM, Chandrasekaran N, Mukherjee A: Studies on Effect of TiO2 Nanoparticles on Growth and Membrane Permeability of Escherichia coli, Pseudomonas aeruginosa, and Bacillus subtilis. Curr Nanosci 2010, 6(4):381-387.

37. Padmavathy N, Vijayaraghavan R: Interaction of $\mathrm{ZnO}$ nanoparticles with microbes-a physio and biochemical assay. J Biomed Nanotechnol 2011, 7(6):813-822.

38. Fang T-T, Li X, Wang Q-S, Zhang Z-J, Liu P, Zhang C-C: Toxicity evaluation of CdTe quantum dots with different size on Escherichia coli. Toxicol In Vitro 2012, 26(7):1233-1239.

39. Kumar A, Pandey AK, Singh SS, Shanker R, Dhawan A: Engineered ZnO and $\mathrm{TiO}(2)$ nanoparticles induce oxidative stress and DNA damage leading to reduced viability of Escherichia coli. Free Radic Biol Med 2011, 51(10):1872-1881.

40. Pan H, Feng J, Cerniglia CE, Chen H: Effects of Orange II and Sudan III azo dyes and their metabolites on Staphylococcus aureus. J Ind Microbiol Biotechno 2011, 38(10):1729-1738. 
41. Pan H, Feng J, He G-X, Cerniglia CE, Chen H: Evaluation of impact of exposure of Sudan azo dyes and their metabolites on human intestinal bacteria. Anaerobe 2012, 18(4):445-453.

42. Sharma V, Shukla RK, Saxena N, Parmar D, Das M, Dhawan A: DNA damaging potential of zinc oxide nanoparticles in human epidermal cells. Toxicol Lett 2009, 185(3):211-218.

43. Zhang Y, Ferguson SA, Watanabe F, Jones Y, Xu Y, Biris AS, Hussain S, Ali SF: Silver nanoparticles decrease body weight and locomotor activity in adult male rats. Small 2013, 9(9-10):1715-1720.

44. Xu H, Heinze TM, Paine DD, Cerniglia CE, Chen H: Sudan azo dyes and Para Red degradation by prevalent bacteria of the human gastrointestinal tract. Anaerobe 2010, 16(2):114-119.

45. Stingley RL, Zou W, Heinze TM, Chen H, Cerniglia CE: Metabolism of azo dyes by human skin microbiota. J Med Microbiol 2010, 59(Pt 1):108-114.

46. Mangoni ML, Papo N, Barra D, Simmaco M, Bozzi A, Di Giulio A, Rinaldi AC Effects of the antimicrobial peptide temporin $L$ on cell morphology, membrane permeability and viability of Escherichia coli. Biochem J 2004, 380(Pt 3):859-865.

doi:10.1186/s12866-014-0222-6

Cite this article as: Pan et al: A comparison of conventional methods for the quantification of bacterial cells after exposure to metal oxide nanoparticles. BMC Microbiology 2014 14:222.

\section{Submit your next manuscript to BioMed Central and take full advantage of:}

- Convenient online submission

- Thorough peer review

- No space constraints or color figure charges

- Immediate publication on acceptance

- Inclusion in PubMed, CAS, Scopus and Google Scholar

- Research which is freely available for redistribution 